\title{
Image Objects Segmentation and Tracking based on Genetic Algorithm Optimized Local Level Set Method with Shape Prior
}

\author{
Aixia Wang, Jingjiao Li*, Zhenni Li, Aiyun Yan \\ College of Information Science and Engineering, Northeastern University, Shenyang, 110819, China
}

\begin{abstract}
The shape prior based level set method is widely used to segment and track objects' contours in images and video sequences. However, such type of method is very slow and easy to fall into local minimum and obtain a wrong matching result. To overcome these issues, this paper presents a novel dynamic local level set method with shape prior. To speed up the local level set method, a genetic algorithm is used to dynamically choose the local region. Secondly, the genetic algorithm is also used to help the evolution process jump out of the local optimum when embedding shape prior into the level set function. The main the main strategy is using genetic algorithm to pre-choose shape priors and estimate the parameters. The experimental results prove the effectiveness and efficiency of the proposed method.
\end{abstract}

Keywords: image segmentation; active contour; level set; genetic algorithm

(Submitted on September 15, 2017; Revised on October 20, 2017; Accepted on October 27, 2017)

(C) 2017 Totem Publisher, Inc. All rights reserved.

\section{Introduction}

Among variation approaches, the level set method has become a popular framework for objects segmentation and tracking in images. The key idea of the level set method is using the zeros level set of a surface to represent the contour of the objects in images. The main advantages of the level set method are that it can naturally obtain closed contours, automatically break and merge regions, and easily embed shape priors into the evolution process. It is especially important when the image background is complex, and the foreground objects are composed by multi-regions. In these situation, popular edge detection and objects segmentation methods such as Canny edge detector [5] or threshold based methods [3] can't yield satisfied results, making the level set method widely used in such situation.

However, there are a series of issues when implementing the level set method. The two most significant issues may be the speed of the level set method and the local optima issue. First, for the basic level set method, during the evolution process of the level set function, each location in the level set function will be updated using partial differential equations. Such situation will be more severe when a local level set method is used [4]. To overcome this issue, parallel algorithms are usually used [6]; however, such type of methods don't solve this problem from the algorithm aspect. Meanwhile, when embedding shape priors into the level set function, in each iteration, partial differential equations will be conducted on the level set function regarding 4 extra parameters, including translation along $x$ and $y$ direction, orientation $\theta$, as well as scale factor $s$, while greatly increasing the computational cost $[1,9,11]$. Second, when intensity distribution is not homogeneous in whole images, a local level set method is usually adopted. However, such type of method is easy converge to a local optimum value. Meanwhile, such issue also happens when embedding shape priors into the level set function, resulting wrong shape matching $[2]$.

To overcome these two issues, a novel dynamic local level set method with shape prior is proposed. Two key insights of this paper are as follows: firstly, the performance and speed of the local level set method is highly related to the local region, 
which depends on the intensity distribution of the region around the current point. Over large and small local region will both reduce the efficiency and effectiveness of the algorithm. In this paper, to speed up the local level set method, a genetic algorithm [8] is used to dynamically choose the local region. Secondly, the genetic algorithm is also used to help the evolution process jump out of the local optimum when embedding shape prior into the level set function. The main strategy is using genetic algorithm to pre-choose shape priors and estimate the parameters such as translation along $x$ and $y$ direction, orientation $\theta$, as well as scale factor $s$, rather than calculate them according to the partial differential equation.

The rest of this paper is organized as follows: Section 2 gives the mathematical background. In Section 3, first the whole framework of this paper is presented, followed by some detail discussion of the key novel ideas of the proposed methods. Section 4 shows the experiment and discussion. Section 5 is the conclusion.

\section{Level set with shape priors}

Originally introduced in the community of computational physics as a means of propagating interfaces, the level set method has become a popular framework for image segmentation. The central idea is to implicitly represent a contour $C$ in the image plane $\Omega \subset \mathbb{R}^{2}$ as the zero-level of an embedding function $\phi: \Omega \rightarrow \mathbb{R}:$

$$
C=\{x \in \Omega \mid \phi(x)=0\}
$$

Rather than directly evolving the contour $C$, one evolves the level set function $\phi$. The two main advantages are that one does not need to deal with control or marker points and the embedded contour is free to undergo topological changes such as splitting and merging, which makes is well-suited for the segmentation of multiple or multiply-connected objects.

Let $\phi$ be the level set function for segmentation, and $\phi_{0}$ be the one embedding a given shape. Both are signed distance functions. Then their shape difference reads as was proposed in [5]:

$$
E_{s}(\phi)=\int_{\Omega}\left(H(\phi)-H\left(\phi_{0}\right)\right)^{2} d x
$$

Theory mentioned above is about the singe shape prior. In [6], given $N$ aligned training samples where $\hat{\phi}_{i}$ is the aligned transformation of $\phi_{i}$, a variational framework can be constructed for the estimation of the Best shape by seeking for the maximum likelihood of the local densities with respect to $(\phi, \sigma)$ :

$$
E_{s}(\phi, \sigma)=-\sum_{i=1}^{n} \iint_{x, y} \log \left[p_{x, y}\left(\hat{\phi}_{i}(x, y)\right)\right] d x d y
$$

where each grid location can be described in the shape model using a Gaussian density function:

$$
p_{x, y}\left(\hat{\phi}_{i}\right)=\frac{1}{\sqrt{2 \pi} \sigma(x, y)} e^{-\frac{\left(\hat{\phi}_{i}-\phi\right)^{2}}{2 \sigma^{2}(x, y)}}
$$

\section{Optimizing Local Level Set with Shape Prior by Genetic Algorithm}

\subsection{The proposed local level set model}

Firstly, we give the proposed level set energy:

$$
E=E_{\text {region }}+\lambda_{1} E_{\text {smooth }}+\lambda_{2} E_{\text {regulation }}+\lambda_{3} E_{\text {shape }}
$$

This representation contains four items: $E_{\text {region }}$ is the energy of the image, basing on the CV model [7], we introduce localizing strategy [4] into it and get:

$$
E_{\text {region }}=\lambda_{4} \int_{\Omega} K H(\phi)\left(I(x)-c_{1}\right)^{2} d x+\lambda_{5} \int_{\Omega} K(1-H(\phi))\left(I(x)-c_{2}\right)^{2} d x
$$

where $c_{1}$ and $c_{2}$ are as follows:

$$
c_{1}(\phi)=\frac{\int_{\Omega} u_{0}(x, y) K H_{\varepsilon}(\phi(t, x, y)) d x d y}{\int_{\Omega} K H_{\varepsilon}(\phi(t, x, y)) d x d y}
$$




$$
c_{2}(\phi)=\frac{\int_{\Omega} u_{0}(x, y) K\left(1-H_{\varepsilon}(\phi(t, x, y))\right) d x d y}{\int_{\Omega} K\left(1-H_{\varepsilon}(\phi(t, x, y))\right) d x d y}
$$

$\mathrm{K}$ is any kind of kernel; in this paper we choose a round 0-1 kernel. With localizing strategy, the model can deal with images with asymmetric grey level.

To overcome the re-initial problem, we introduced the following item proposed by $\mathrm{Li}$ in [4].

$$
E_{\text {regulation }}=\int \frac{1}{2}(|\nabla \phi(x)|-1)^{2} d x
$$

As an effective smoother, Length term is very popular in level set represents, in this paper, the following item is used:

$$
E_{\text {regulation }}=\int_{\Omega}|\nabla H(\phi(x, y))| d x d y
$$

Now we discuss about the $E_{\text {shape }}$. Firstly, the $E_{\text {shape }}$ is a sum of weighted kernel density estimation. Employ $d(\alpha, \beta)$ to denote the distance of $\alpha$ and $\beta$ in kernel space, the $E_{\text {shape }}$ can be written:

$$
E_{\text {shape }}=\frac{\sum_{i=1}^{N} \alpha_{i} d\left(H(\phi), H\left(\phi_{i}\right)\right)}{2 \sigma^{2} \sum_{i=1}^{N} \alpha_{i}}
$$

which simply induces a force in direction of each training shape $\phi_{i}$ weighted by the factor:

$$
\alpha_{i}=\exp \left(-\frac{1}{2 \sigma^{2}} d\left(H(\phi), H\left(\phi_{i}\right)\right)\right)
$$

Taking translation by $\mu$, rotation by an angle $\theta$ and scaling by the $s$ of shape into account:

$$
d\left(\phi, \phi_{0}\right)=\int_{\Omega}\left(H\left(\phi\left(s R_{\theta}(x-\mu)\right)\right)-H\left(\phi_{0}\right)\right)^{2} d x
$$

\subsection{Dynamic localized active contour with shape prior}

Firstly, it needs to encode the considered parameters into the genes in genetic algorithm. In the proposed model, each shape energy contains: the position $\mathrm{P}$ with two parameters $(\mathrm{x}, \mathrm{y})$, scale $\mathrm{S}$ and angle $\mathrm{A}$. We also consider the situation of the shape reversal, which can be denoted by a binary number $\mathrm{R}$, in which value -1 represents that the object's shape is reversal to the shape prior while value +1 represents the opposition. All of above parameters are PSAR for short. To improve the rate and accuracy of the computation, the initial value of these parameters needs to determine. We can estimate the PSA as follows:

$$
\begin{gathered}
S_{0}=\sum_{x} \sum_{y} H(\phi(x, y)) \\
P_{0}\left(x_{c}, y_{c}\right)=\left(\frac{m_{10}}{S}, \frac{m_{01}}{S}\right)
\end{gathered}
$$

where $m_{10}=\sum_{x} \sum_{y} x H(\phi(x, y)), m_{01}=\sum_{x} \sum_{y} y H(\phi(x, y))$.

We can also calculate the direction of the shape:

$$
A_{0}=\frac{\arctan \left(\frac{b}{a-c}\right)}{2}
$$

where $a=\frac{m_{20}}{S}-x_{c}^{2}, b=2\left(\frac{m_{11}}{S}-x_{c} y_{c}\right), c=\frac{m_{02}}{S}-y_{c}^{2}$.

But it should be noticed that when calculating S, P and A of the object, as the contour of the object is still in evolution process, all of these parameters are imprecise, which are only the estimation of the object. The ranges of value of these parameters are: $\mathrm{S}$ is in the range of $\left[0.8 S_{0}, 1.2 S_{0}\right] ; P_{x 0}$ is in the range of $\left[P_{x 0}-\sqrt{S_{0}}, P_{x 0}+\sqrt{S_{0}}\right] ; P_{y 0}$ is in the range of $\left[P_{y 0}-\sqrt{S_{0}}, P_{y 0}+\sqrt{S_{0}}\right] ; \mathrm{A}$ is in the range of $\left[A_{0}-\frac{\pi}{2}, A_{0}+\frac{\pi}{2}\right] ; \mathrm{R}$ is either +1 or -1 . 
In the calculating process, we always make two genes' PSAR in each subgroup to be $\left\{P_{0}, S_{0}, A_{0}, 1\right\}$ and $\left\{P_{0}, S_{0}, A_{0},-1\right\}$ respectively. Actually, if we only have one gene with value of $\left\{P_{0}, S_{0}, A_{0}, 1\right\}$, our model is equal to [4].

Fitness of each gene is equal to its corresponding shape energy, and there will be two stop criterions: one is the predefined max iterative number Ms, and the other is that if the best gene's fitness is unchanged in Mp steps; then, the evolution process should be over.

The whole population of genes in GA will be divided into $\mathrm{N}$ subgroups, which is the same as the number of shape priors. The shape prior is not similar to the current object shape, which is indicated as follows:

$$
\text { fitness }_{i}>\text { fitness }_{\text {mean }}+3 * \text { fitness }_{\sigma}
$$

The $i$-th shape prior will be excluded and the corresponding subgroup will be deleted.

\section{Experiments}

In this section, we conduct two groups of experiments. In the first group, we test the proposed dynamic local level set method on four images. As comparisons, we also test the segmentation performance of three other level set method, namely, Kasmin [3], Li [4], and Yang [10]. In the second groups, we compare the proposed dynamic local level set method with shape prior with Qin [7].

\subsection{Experiments of the dynamic local level set method}

In this experiment, we test the proposed dynamic local level set method on four images. As comparisons, we also test the segmentation performance of three other level set method, namely, Li [4], Kasmin [3], and Yang [10]. Li [4] is the classic local level set method, Kasmin [3], and Yang [10] are state-of-the-art method. The parameters used in this experiment are as follows: the number of individual is 100 , the max step is 30 , the max unchanged step is 5 , the crossover rate is 0.3 . Parameters in level set: $\lambda_{1}=1, \lambda_{3}=1, \lambda_{4}=1, \lambda_{5}=1, \lambda_{2}=255 * 255 * 0.005$. The radius of round $0-1$ kernel is 5 . We used parameters of other methods as recommended in their papers. The platform is Matlab 2010b, CPU is i7, memory is 8GB. The results are shown in Figure1 Figure4, and the running time are shown in Table 1.

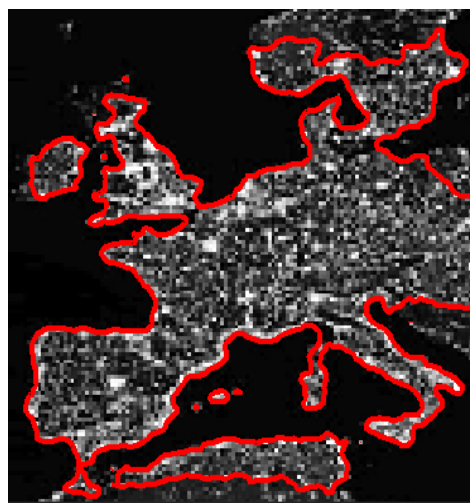

(a)

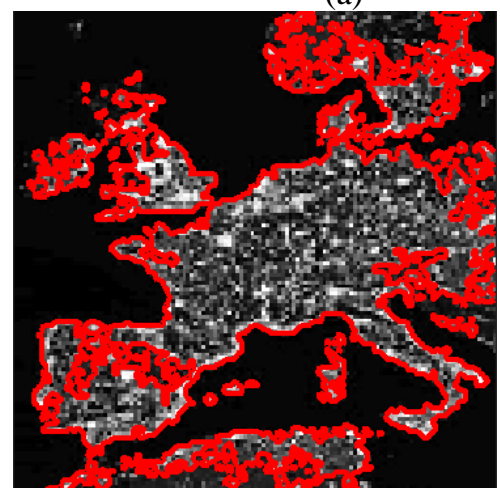

(c)

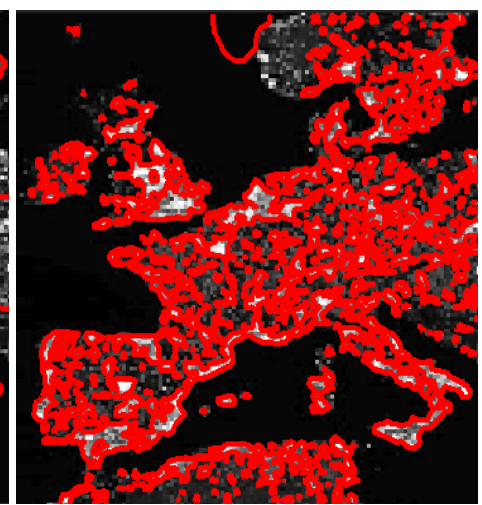

(b)

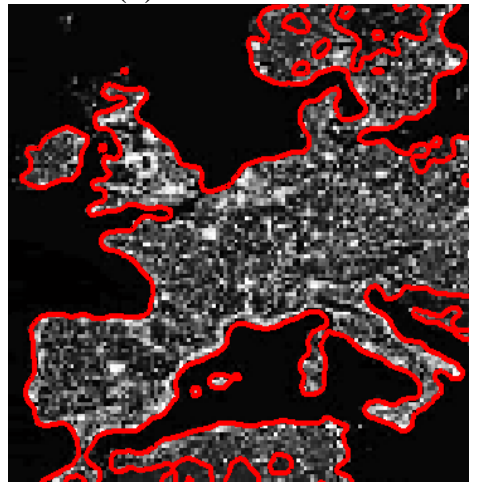

(d)

Figure 1. Comparions on Image 1 


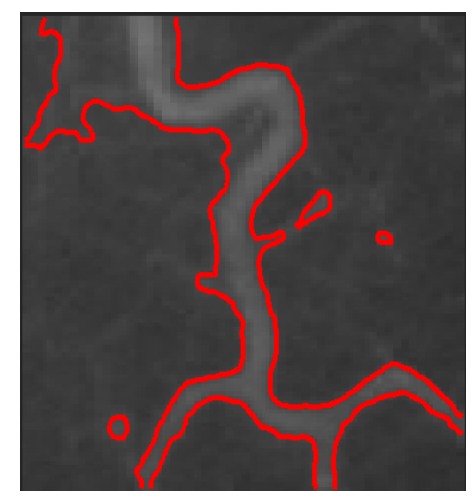

(a)

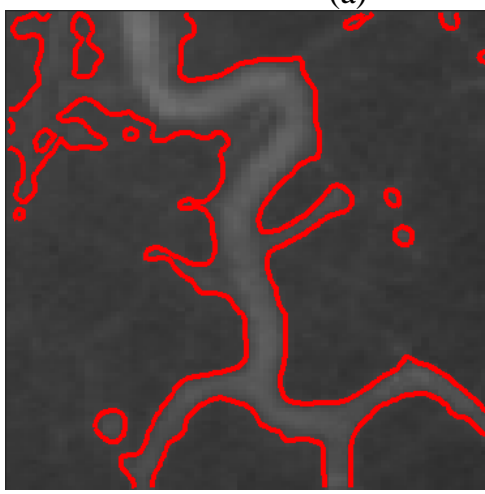

(c)

Figure 2. Comparions on Image 2

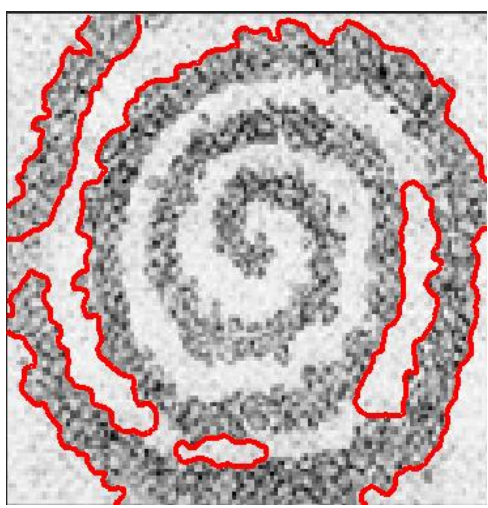

(a)
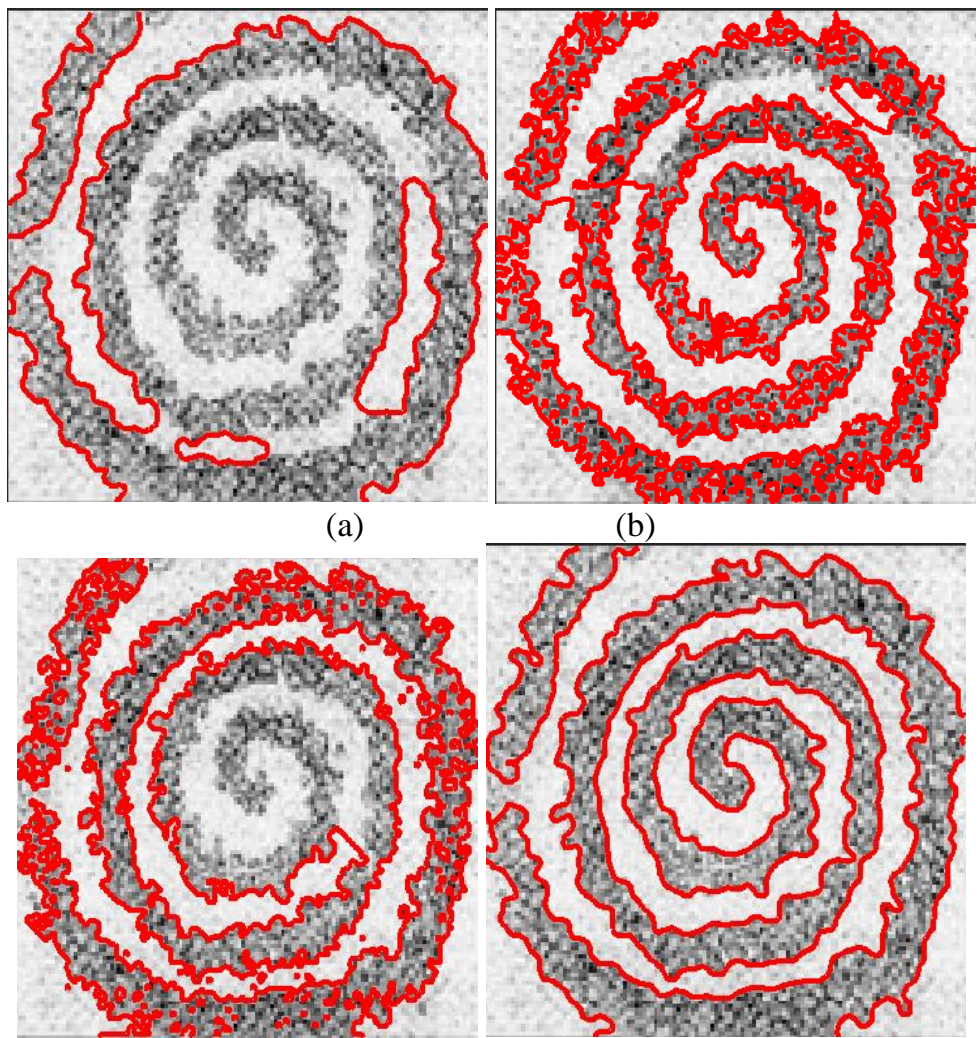

(b)

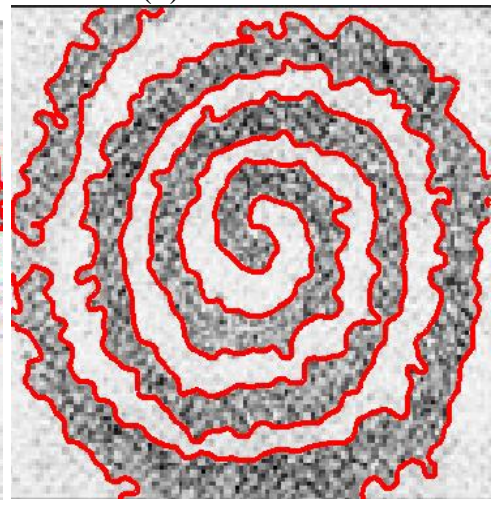

(d)

Figure 3. Comparions on Image 3 


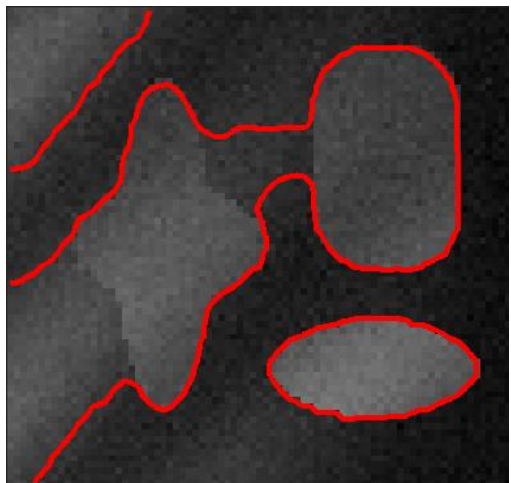

(a)

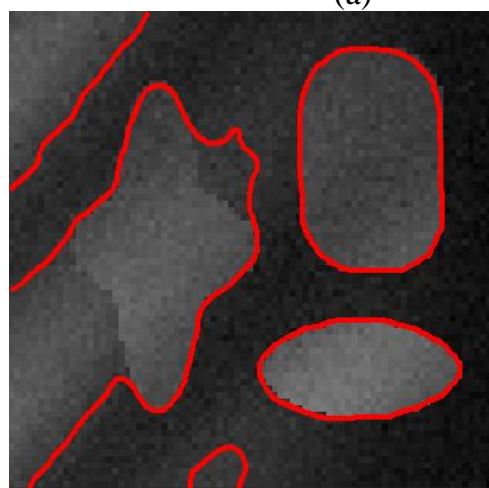

(c)

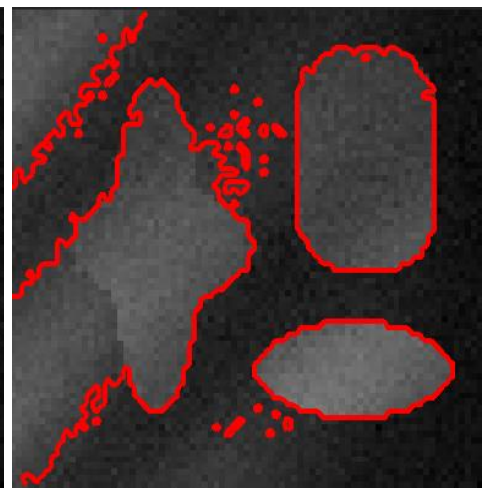

(b)

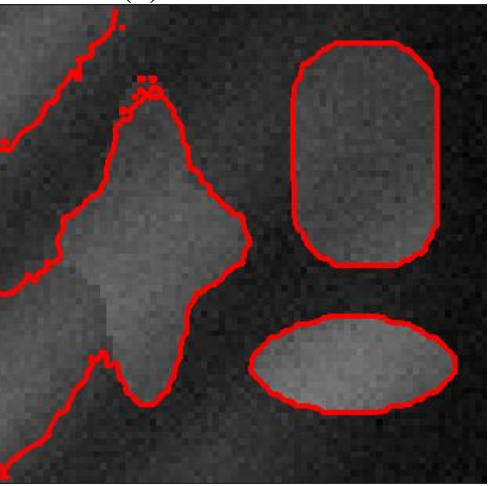

(d)

Figure 4. Comparions on Image 4

Table 1. Running time of methods on different images

\begin{tabular}{|c|c|c|c|c|c|}
\hline \multicolumn{2}{|c|}{ Method } & Li[4] & Kasmin [3] & Yang[10] & Our method \\
\hline \multirow{3}{*}{ Running Time } & Image 1 & $248 \mathrm{~s}$ & $211 \mathrm{~s}$ & $68 \mathrm{~s}$ \\
\cline { 2 - 6 } & Image 2 & $253 \mathrm{~s}$ & $228 \mathrm{~s}$ & $11 \mathrm{~s}$ \\
\cline { 2 - 6 } & Image 3 & $264 \mathrm{~s}$ & $225 \mathrm{~s}$ & $138 \mathrm{~s}$ \\
\cline { 2 - 6 } & Image 4 & $217 \mathrm{~s}$ & $196 \mathrm{~s}$ & $98 \mathrm{~s}$ & $62 \mathrm{~s}$ \\
\hline
\end{tabular}

As shown in Figure 1 through Figure 4, compared with Kasmin[3] and Yang[10], the segmentation result of Li[4] and our method are much better. The main reason is that both of Li[4] and our method use local level set method, which can overcome the issue of uneven distribution of the image intensity. However, the proposed method is much faster than Li[4], due the method of dynamic local level set method.

\subsection{Experiments of the dynamic local level set method with shape prior}

In this experiment, we test the proposed dynamic local level set method with shape prior on the dataset. As comparisons, we also test the segmentation performance of a state-of-the-art method Qin[7]. The parameters used in this experiment are as follows: the number of individual is 100 , the max step is 30 , the max unchanged step is 5 , the crossover rate is 0.3 . Parameters in level set: $\lambda_{1}=1, \lambda_{3}=1, \lambda_{4}=1, \lambda_{5}=1, \lambda_{2}=255^{*} 255^{*} 0.005$. The radius of round $0-1$ kernel is 5 . We used parameters of other methods as recommended in their papers. The platform is Matlab 2010b, CPU is i7, memory is 8GB.

Figure 5 shows parts of shape priors using in this test.

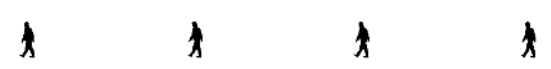

Figure 5. Shape priors

Figure 6 shows the tracking results of the experiment. In the first column, the person in the video sequence faces left, which is the same as the shape priors. But in the second column, the person in the video sequence faces right, which is opposite with the shape priors. In some previous framework of segmentation with shape priors, it can hardly be deal with. In the proposed algorithm, as the inversion parameter was considered, we still can track the object in this situation. Actually, any affine parameter can be added into the proposed model by appending the parameter into the gene. In all figures in Figure 6 , it can be seen that even the object was partly occlusive, the algorithm can still segment the object correctly. 


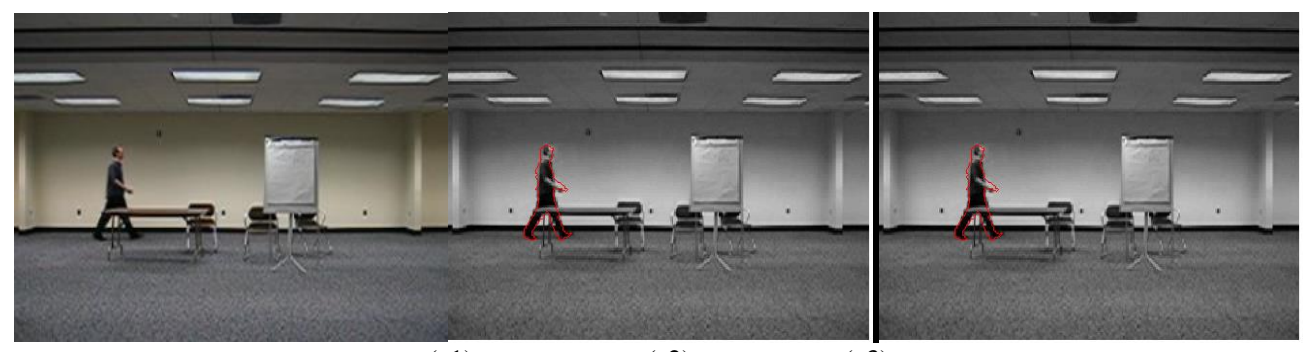

(a1)

(a2)

(a3)

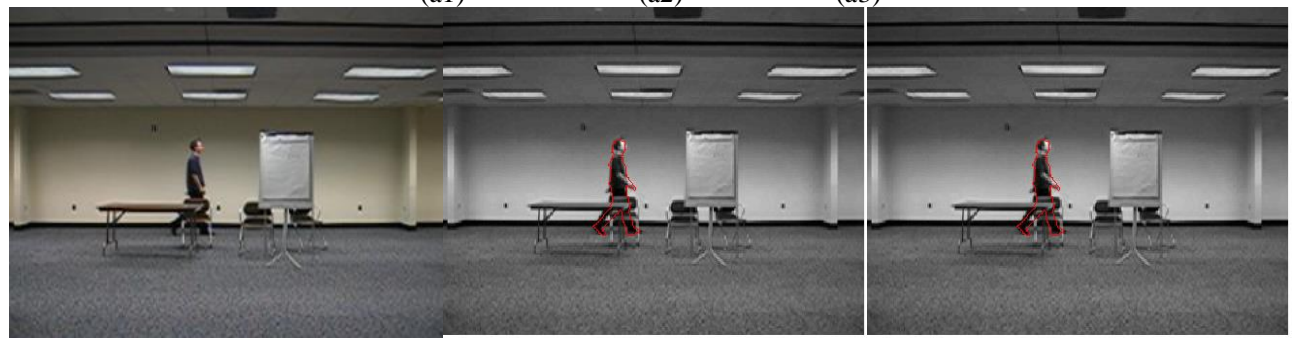

(b1)

(b2)

(b3)

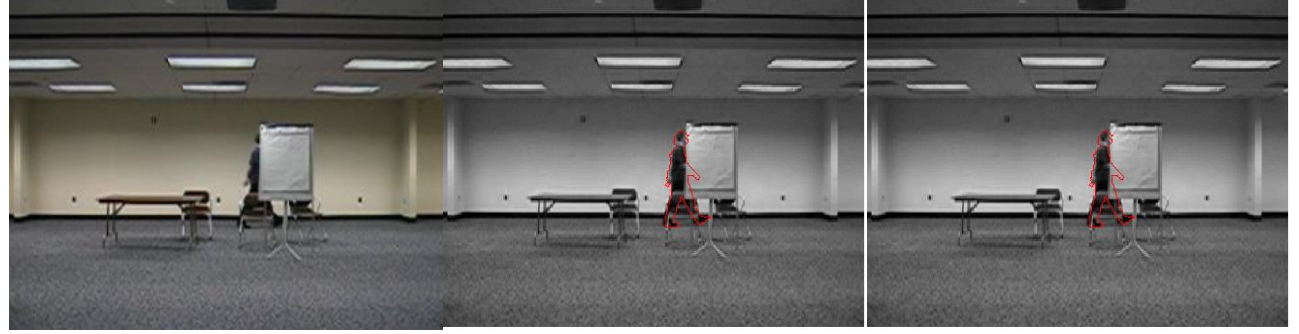

(c1)

(c2)

(c3)

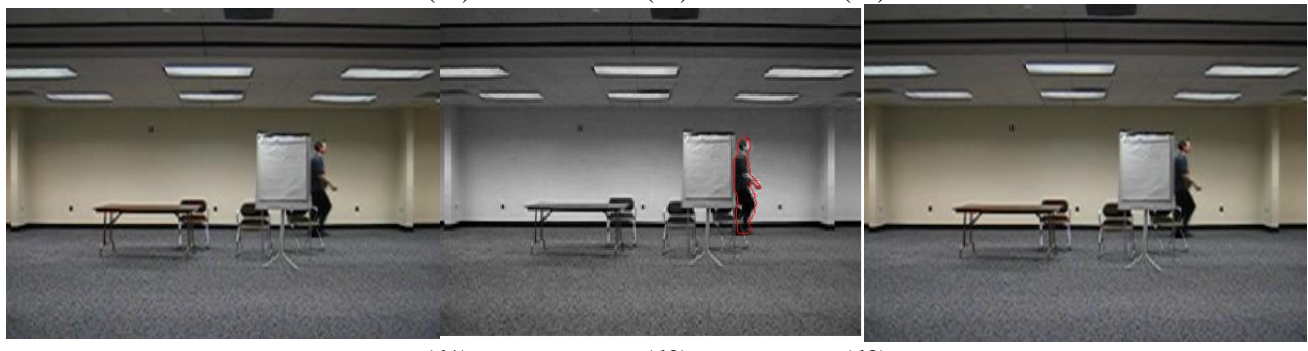

(d1)

(d2)

(d3)

Figure 6. Tracking results

Table 2. Running time of methods on different images

\begin{tabular}{|c|c|c|c|}
\hline \multicolumn{2}{|c|}{ Method } & Qin [7] & Our method \\
\hline \multirow{3}{*}{ Running Time } & Image 1 & $763 \mathrm{~s}$ & $112 \mathrm{~s}$ \\
\cline { 2 - 4 } & Image 2 & $768 \mathrm{~s}$ & $108 \mathrm{~s}$ \\
\cline { 2 - 4 } & Image 3 & $754 \mathrm{~s}$ & $116 \mathrm{~s}$ \\
\cline { 2 - 4 } & Image 4 & $217 \mathrm{~s}$ & $133 \mathrm{~s}$ \\
\hline
\end{tabular}

As shown in Figure 6, for the first three images, both Qin [7] and our method can obtain correct tracking result. However, in the last image, Qin [7] completely lost the object, while our method can still obtain correct tracking result. The main reason is that the genetic algorithm used in our method can search more regions than Qin [7], which only uses partial differential equation to evolution the location of the object.

Meanwhile, from Table 2, it can be seen that our method is much faster than Qin [7], as our method can search the situation translation, orientation and scale more quickly than the differential equation used by Qin [7].

\section{Conclusions}

This paper presents a novel dynamic local level set method with shape prior. To speed up the local level set method, a genetic algorithm is used to dynamically choose the local region. Secondly, the genetic algorithm is also used to help the evolution process jump out of the local optimum when embedding shape prior into the level set function. The main the main 
strategy is using genetic algorithm to pre-choose shape priors and estimate the parameters. The experimental results prove the effectiveness and efficiency of the proposed method.

\section{References}

1. D.Cremers, S.J.Osher, and S.Soatto, "Kernel density estimation and intrinsic alignment for shape priors in level set segmentation," International Journal of Computer Vision, vol.69, no.3, pp. 335-351, 2006

2. S.Dambreville, Y.Rathi, and A.Tannenbaum, "A framework for image segmentation using shape models and kernel space shape priors, " IEEE Transactions on Pattern Analysis and Machine Intelligence, vol.30, no.8, pp. 1385-1399, 2008

3. F.Kasmin, A.Abdullah, and A. S.Prabuwono, "Ensembles of Normalization Techniques to Improve the Accuracy of Otsu Method," Applied Mathematical Sciences, vol.9, pp. 1565-1578, 2015

4. C.M.Li, C.Y.Kao, J.C.Gore, and Z.H.Ding, "Minimization of Region-Scalable Fitting Energy for Image Segmentation," IEEE Trans. Image Processing, vol.17, no.10, pp. 1940-1949, 2008

5. W.McIlhagga, "The Canny Edge Detector Revisited, " International Journal of Computer Vision, vol.91, no.3, pp.251-261, 2011

6. M.Pereyra., H.Batatia, and S.McLaughlin, "Exploiting information geometry to improve the convergence of nonparametric active contours, " IEEE Transactions on Image Processing, vol.24, no.3, pp. 836-845, 2015

7. X.J.Qin, X.L.Li, Y.Liu, H.B.Lu, and P.K.Yan, "Adaptive Shape Prior Constrained Level Sets for Bladder MR Image Segmentation," IEEE Journal of Biomedical and Health Informatics, vol.18, no.5, pp. 1707-1716, 2014

8. M.Thakur, S.S.Meghwani, and H.Jalota, "A modified real coded genetic algorithm for constrained optimization Applied Mathematics and Computation, " Elsevier, vol.235, pp. 292-317, 2014

9. B.Wang, X.B.Gao, J.Li, X.L.Li, D.Tao, D.C.Tao, "A level set method with shape priors by using locality preserving projections," Neurocomputing, vol.170, pp. 188-200, 2015

10. X.Yang, X.B.Gao, D.C.Tao, X.L.Li, J.Li, "An Efficient MRF Embedded Level Set Method for Image Segmentation," IEEE Transactions on Image Processing,vol. 24, no.1, pp.9-21, 2015

11. Y.Yuan, C.J.He, "Adaptive active contours without edges, ” Mathematical and Computer Modelling, vol.55, pp. 1705-1721, 2012 